Supplemental Information (SI)

\title{
Symmetrical conjugated molecular additive for defect passivation and charge transfer bridge in perovskite solar cells
}

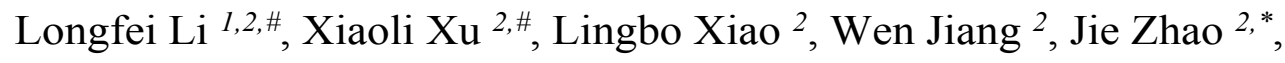

Xiangqiang Kong ${ }^{1, *}$, Guifu Zou ${ }^{1,2, *}$

1 School of Mechanical and Electronic Engineering, Shandong University of Science and Technology, Qingdao. 266590, China.

${ }^{2}$ College of Energy, Soochow Institute for Energy and Materials Innovations, and Key Laboratory of Advanced Carbon Materials and Wearable Energy Technologies. Soochow University, Suzhou, 215000, China.

\# These authors contributed equally to this work.

E-amil: jzhao@suda.edu.cn; xqkong@sdust.edu.cn; zouguifu@suda.edu.cn. 


\section{Experimental Section}

Materials: Dimethylformamide (DMF), dimethyl sulfoxide (DMSO), chlorobenzene and acetonitrile were purchased from Sigma Aldrich. Formamidinium iodide (FAI), lead iodine $\left(\mathrm{PbI}_{2}\right)$, methylammonium bromide $(\mathrm{MABr})$, lead bromide $\left(\mathrm{PbBr}_{2}\right)$, methylammonium $\quad$ chloride $\quad(\mathrm{MACl}), \quad 2,20,7,70$-tetrakis[N,N-di(4methoxyphenyl)amino]-9,90-spirobifluorene (Spiro-OMeTAD), bis(trifluoromethanesulfonyl)imide lithium salt (Li-TFSI), 4-tert-butylpyridine (TBP), [6,6]-Phenyl C61 butyric acid methyl ester (PCBM), poly(3,4-ethylenedioxythiophene):poly(styrene sulfonate) (PEDOT:PSS) were all purchased from Xi'an Polymer Light Technology in China. Cobalt(III) FK209 bis(trifluoromethylsulfonyl)imide salt (FK209) was purchased from Greatcell Solar Materials Pty Ltd. 4,4'-Bipyridyl (BPY[4,4]) was purchased from Energy Chemical, 2,2'-bipyridyl (BPY[2,2]) and 4-cyanopyridine (CNP) were purchased from Jiuding Chemical, purchased from Jiuding Chemical. All the materials were directly used without further purification.

Device fabrication: Fluorine-doped tin oxide (FTO) substrates were successively cleaned with deionized water, ethanol, acetone and Isopropanol for $10 \mathrm{~min}$ in an ultrasonicator and treated using UV-ozone for $20 \mathrm{~min}$ before use. The device configuration is fluorine-doped tin oxide (FTO)/compact $\mathrm{TiO}_{2} /$ perovskite/ SpiroMeOTAD/Au. A 10 vol\% titanium diisopropoxide bis(acetylacetonate) solution in ethanol was spin-coated on the substrate at 3,000 r.p.m. for 30 s. then annealing in ambient air at $500{ }^{\circ} \mathrm{C}$ for $30 \mathrm{~min}$ and cool to ambient temperature to form the compact $\mathrm{TiO}_{2}$ layer. After that, a perovskite solution of formamidinium lead iodide $\left(\mathrm{FAPbI}_{3}\right)$ : methylammonium lead bromide ( $\left.\mathrm{MAPbBr}_{3}\right)$ : methylammonium chloride (MACl) (889 mg: $33 \mathrm{mg}: 33 \mathrm{mg}$ in $1 \mathrm{~mL}$ DMF/DMSO mixed solvent with 8:1 v/v) was deposited by two consecutive spin-coating steps of 1,000 r.p.m. and 4,500 r.p.m. for $5 \mathrm{~s}$ and $15 \mathrm{~s}$, respectively. During the second spin coating, anti-solvent chlorobenzene was evenly dropped onto the substrate after 8 seconds. The perovskite films were immediately transferred on a uniform temperature hotplate at $155^{\circ} \mathrm{C}$ for $15 \mathrm{~min}$. For BPY $[4,4]$ addition, the BPY[4,4] solution dissolved in DMSO was added into the perovskite 
solution with different molar ratio $(0.05,0.15,0.3,0.5,1 \mathrm{~mol} \%$ with respect to $\mathrm{Pb}$ atom $)$ and spin-coated on the substrate just like the fabrication of perovskite films. Other additives were similar to the above addition. After the fabrication of perovskite film, a hole-transport layer, Spiro-OMeTAD (72.3 $\mathrm{mg} \mathrm{mL}^{-1}$ dissolved in chlorobenzene) solution with standard additives of $35 \mu \mathrm{L} \mathrm{Li-TFSI} \mathrm{(} 260 \mathrm{mg} \mathrm{mL}^{-1}$ solution in acetonitrile), $17 \mu \mathrm{L}$ FK209 (165 mg mL $\mathrm{m}^{-1}$ solution in acetonitrile) and $30 \mu \mathrm{L}$ TBP was spin-coated on the perovskite film at 3,000 r.p.m. for 20 s. Finally, Au electrode of $80 \mathrm{~nm}$-thick was deposited by thermal evaporation using a shadow mask.

Characterization: The UV-vis absorbance spectra of the control and PBY[4,4] based perovskite films (0-1 mol\%) were measured on an UV-vis spectrophotometer (Lambda 750S, USA). The steady-state photoluminescence (PL) under an excitation at $535 \mathrm{~nm}$ and time-resolved PL (TRPL) spectra were measured by Horiba fluorescence spectrophotometer (Fluoromax-4, France). The photocurrent density-voltage (J-V) curves of the perovskite solar cells (PSCs) were monitored using a Keithley Model (2400 SourceMeter, USA) under AM 1.5 solar simulator irradiation of $100 \mathrm{~mW} / \mathrm{cm}^{2}$ (XES-300M2, Japan). The incident photon-to-current conversion efficiency (IPCE) spectra of the PSCs were measured using quantum efficiency measurement system (QE-R3018, Taiwan, China). The surface morphology and cross-sectional images of perovskite films were captured using a scanning electron microscope (SEM, Hitachi SU8010, Japan). The crystalline structures of perovskite films were characterized by an X-ray diffractometer (XRD, D8 Advance, German). The elemental composition of the perovskite films was monitored with the $\mathrm{Al} \mathrm{K} \alpha \mathrm{X}$-ray as the excitation source, and the ultraviolet photoelectron spectroscopy (UPS) spectra were recorded with hv of 21.22 $\mathrm{eV}$ on an X-ray photoelectron spectrometer (XPS, Escalab 250Xi, USA), respectively. 

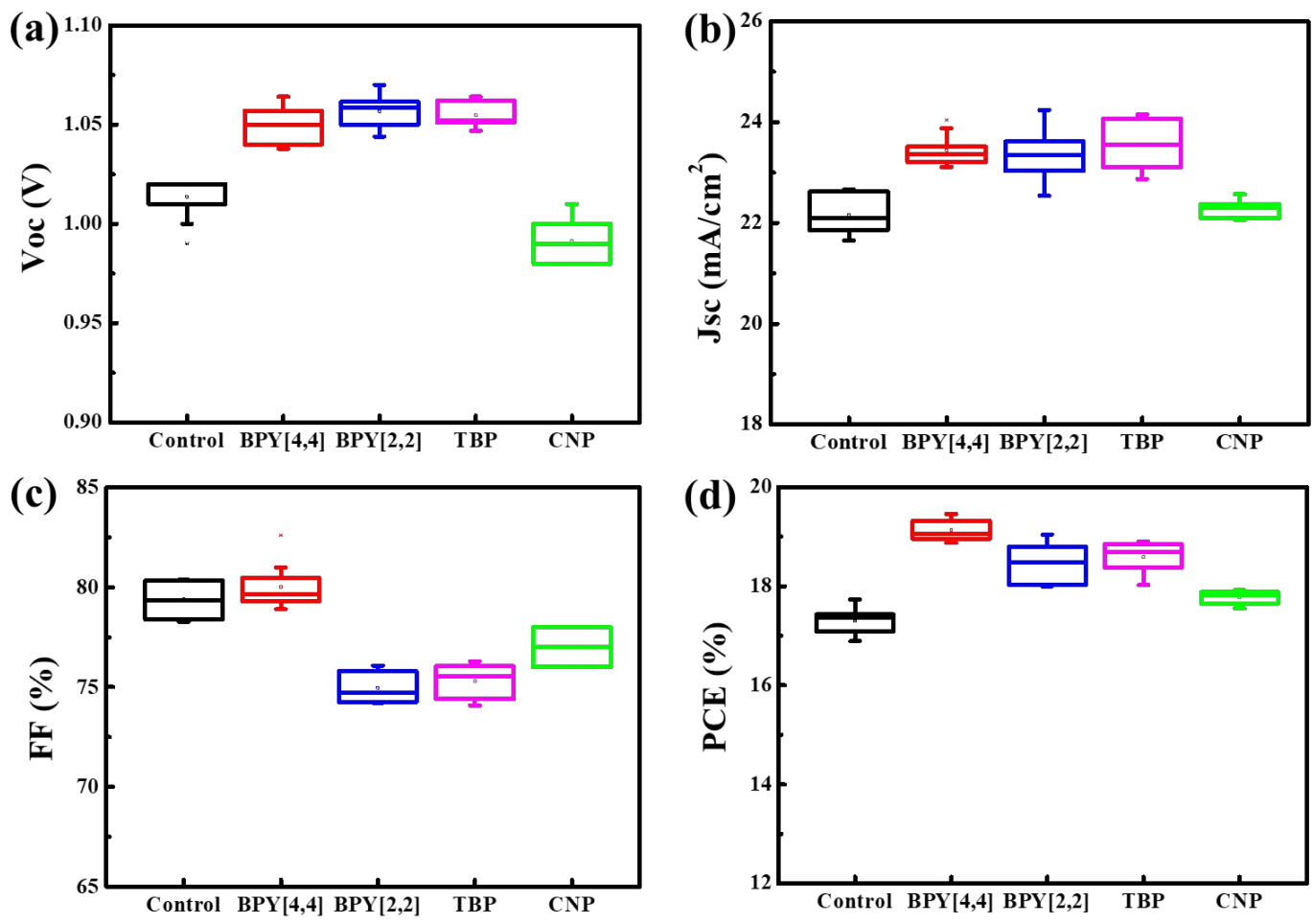

Figure S1. photovoltaic parameter variation of perovskite solar cells with control and different additives containing BPY[4,4], BPY[2,2], TBP and CNP at $0.15 \mathrm{~mol} \%$, respectively. 

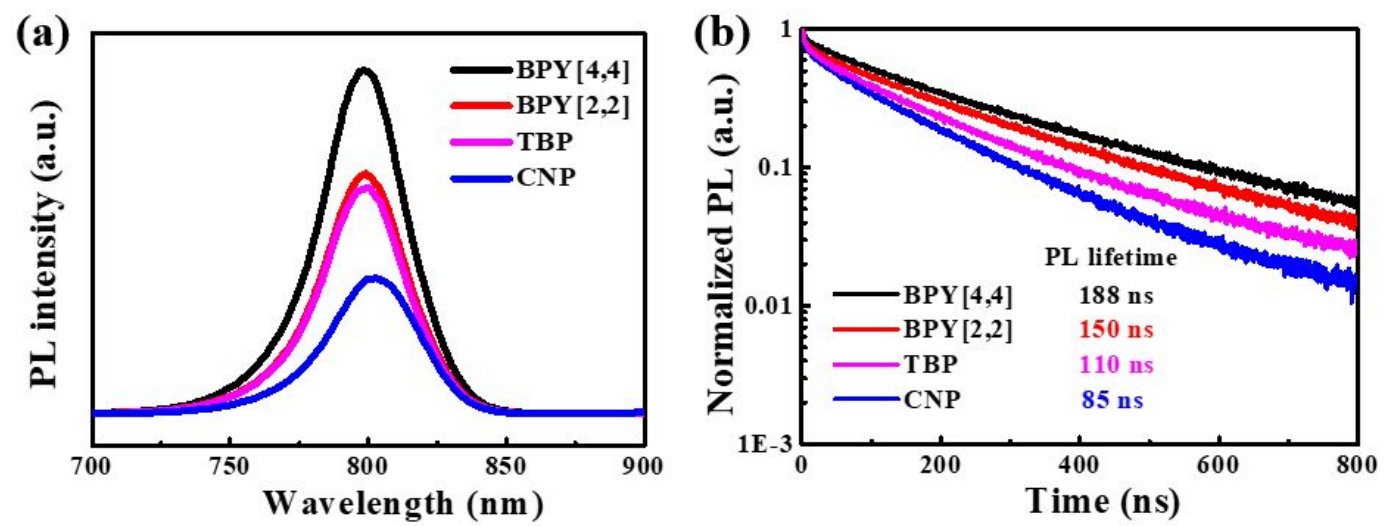

Figure S2. (a) Steady-state PL spectra for perovskite films with different additives containing BPY[4,4], BPY[2,2], TBP and CNP at $0.15 \mathrm{~mol} \%$, respectively. 

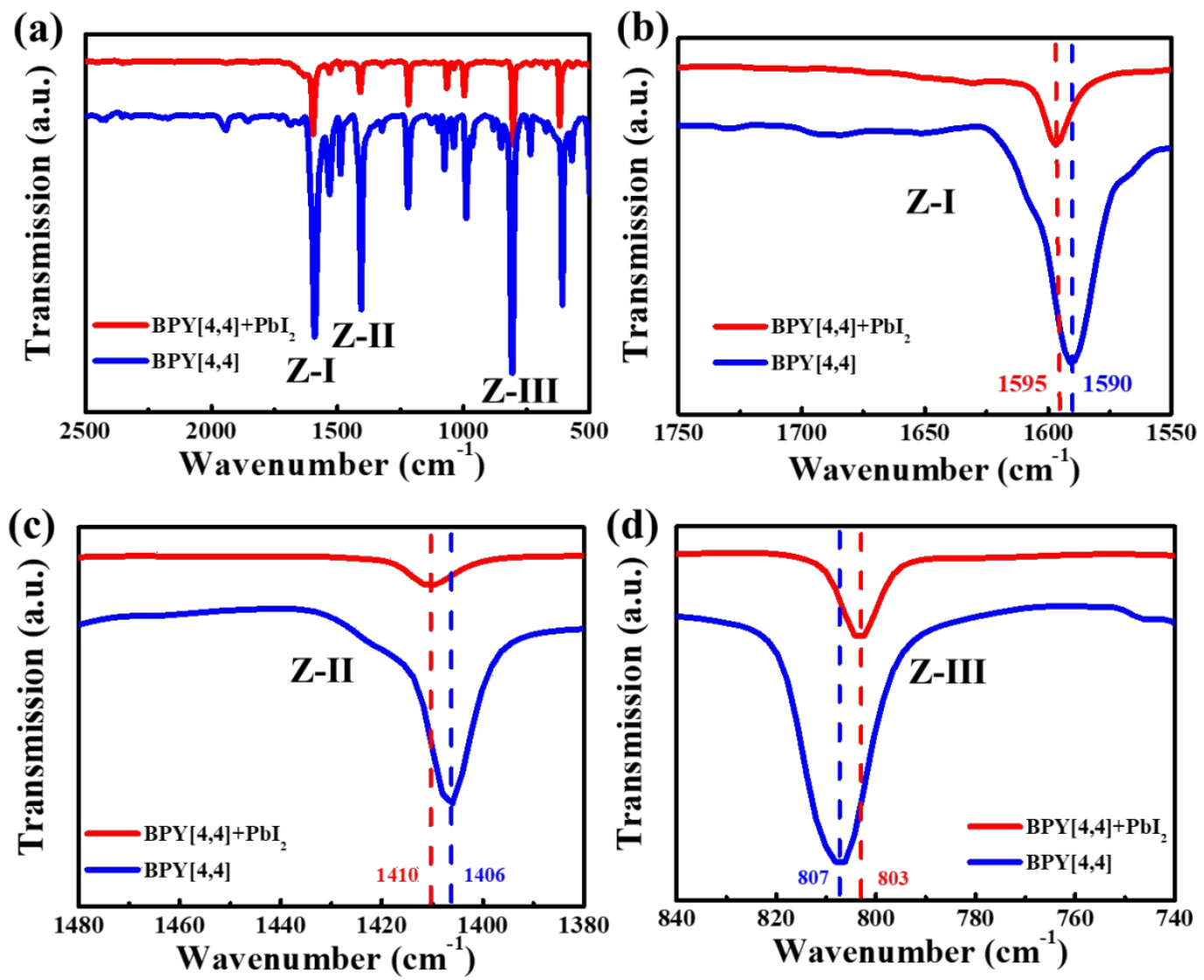

Figure S3. (a) FTIR spectra of BPY $[4,4]$ and BPY $[4,4]-\mathrm{PbI}_{2}$ films. (b)

Enlarged spectral zones Z-I, Z-II and Z-III, respectively. 


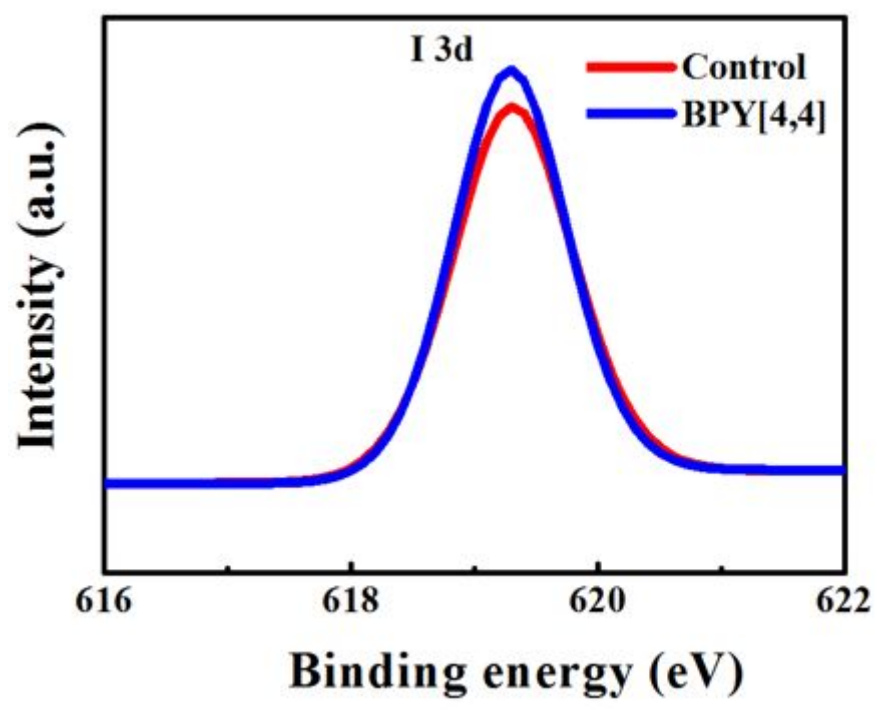

Figure S4. XPS spectra of I 3d core levels for the perovskite layers with or without BPY $[4,4]$ additive. 
(a)

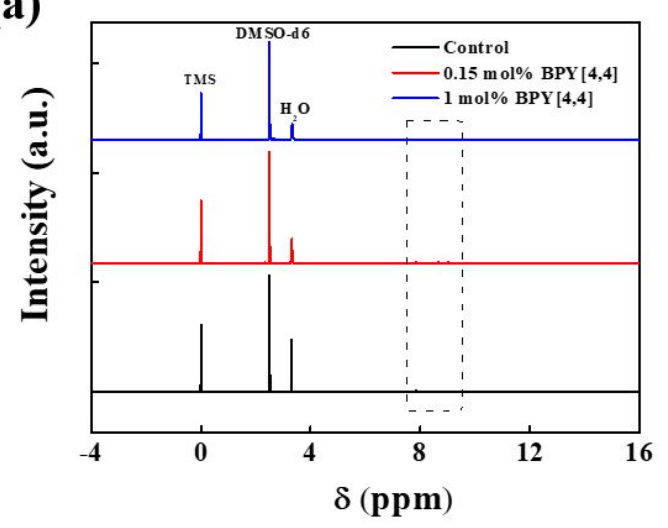

(b)

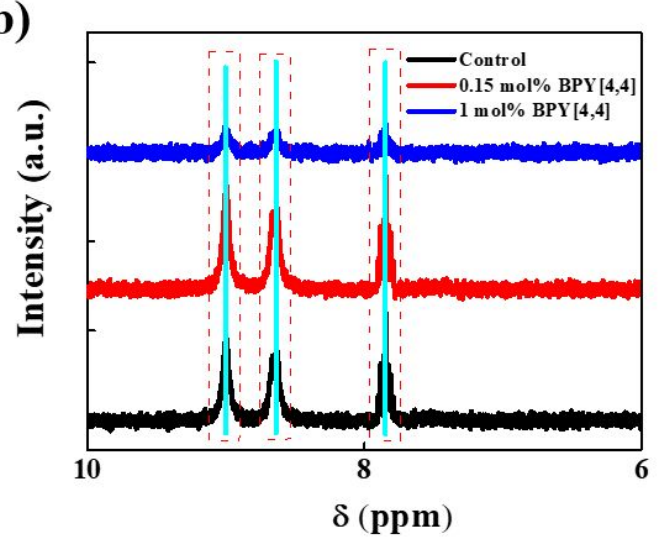

Figure S5. The corresponding ${ }^{1} \mathrm{H}$ NMR showed that the signal changes of control perovskite and different concentrations of BPY $[4,4]$ passivated perovskite, the solution is Dimethyl sulfoxide- $\mathrm{d}_{6}\left(\right.$ DMSO- $\left.\mathrm{d}_{6}\right)$. 

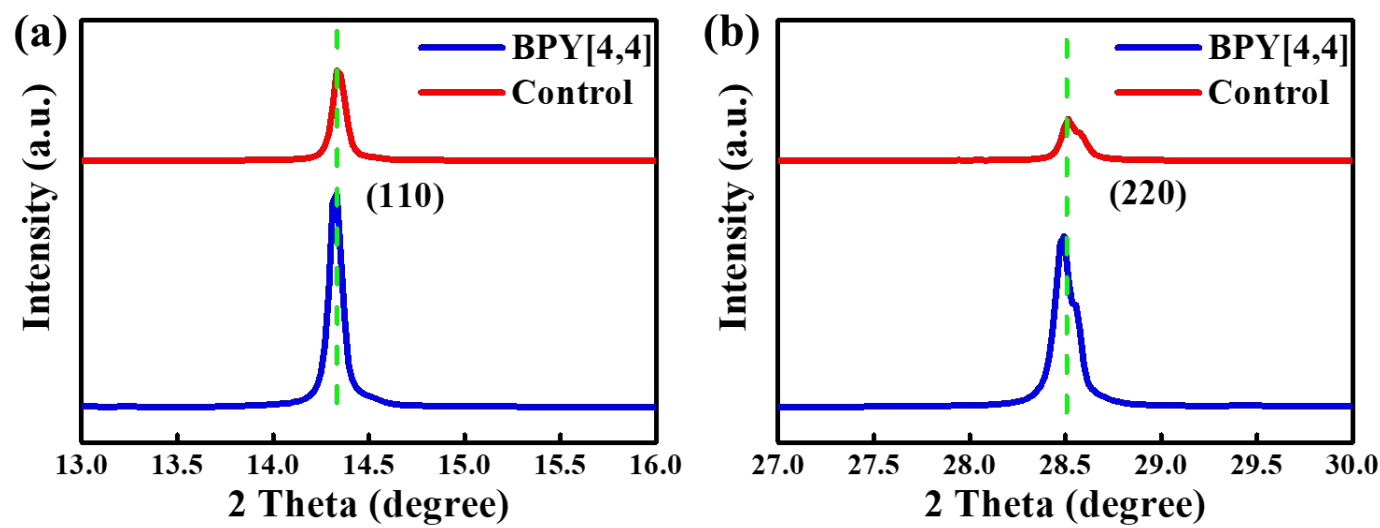

Figure S6. $(\mathrm{a}, \mathrm{b})$ Magnified XRD patterns for control film and BPY $[4,4]$ added perovskite films at (100) and (220) orientation peaks. 

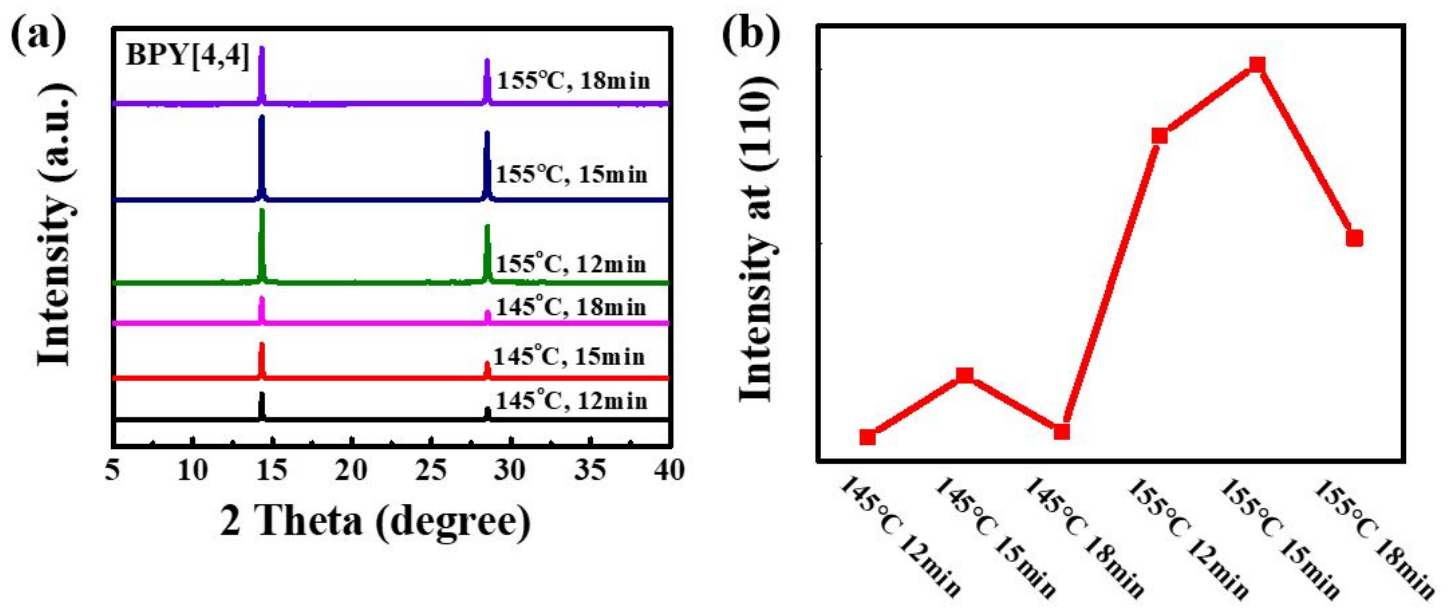

Figure S7. $(a, b)$ Optimization of annealing temperature and time on the (100) peak intensity variation at $13.95^{\circ}$. 


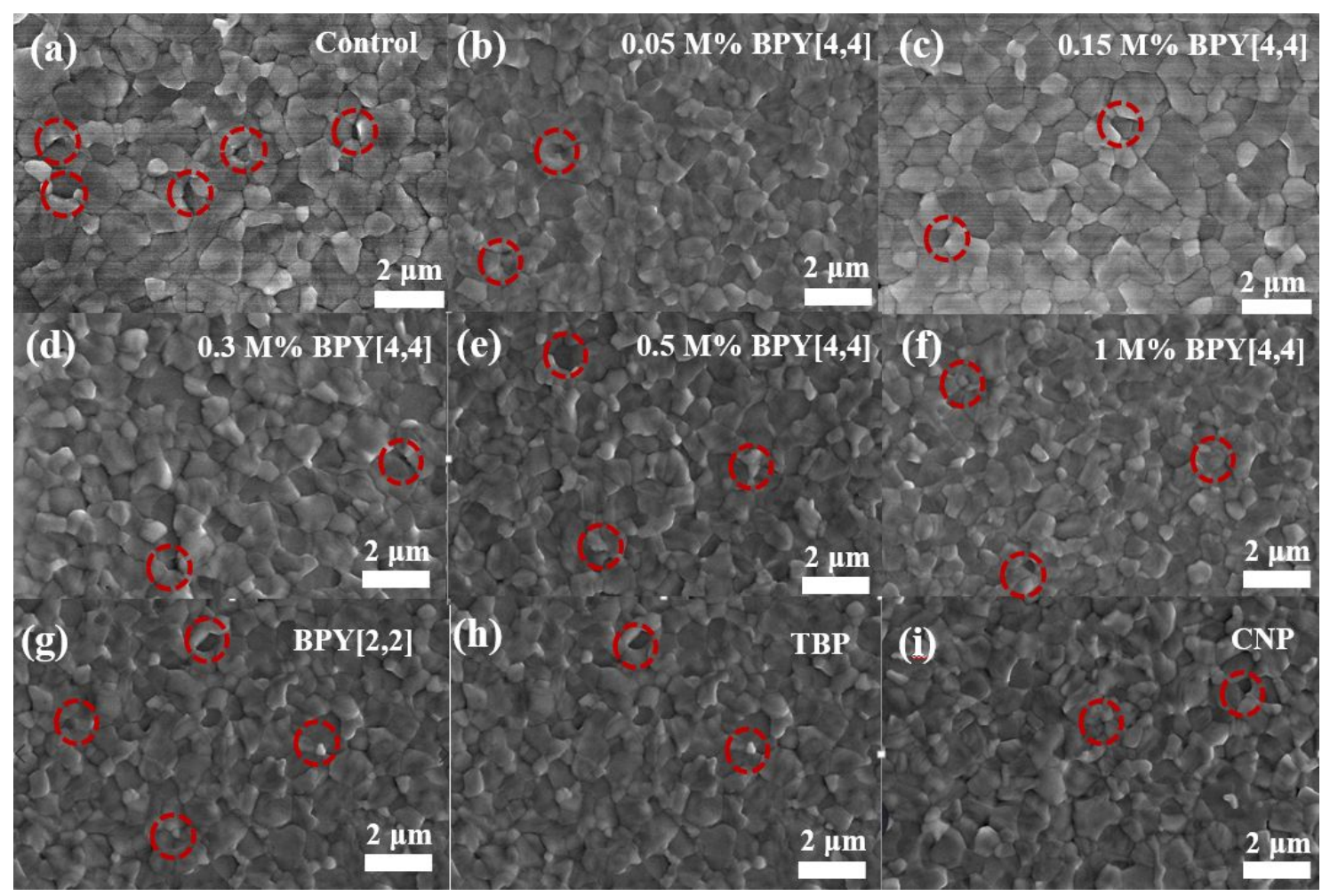

Figure S8. SEM images of different additives and BPY $[4,4]$ at different concentrations. (a) Control (b) $0.05 \mathrm{M} \%$ BPY $[4,4]$ (c) $0.15 \mathrm{M} \%$ BPY $[4,4]$ (d) $0.3 \mathrm{M} \% \mathrm{BPY}[4,4]$ (e) $0.5 \mathrm{M} \% \mathrm{BPY}[4,4]$ (f) $1 \mathrm{M} \% \mathrm{BPY}[4,4](\mathrm{g})$ BPY $[2,2]$ (h) TBP (i) CNP 
(a)
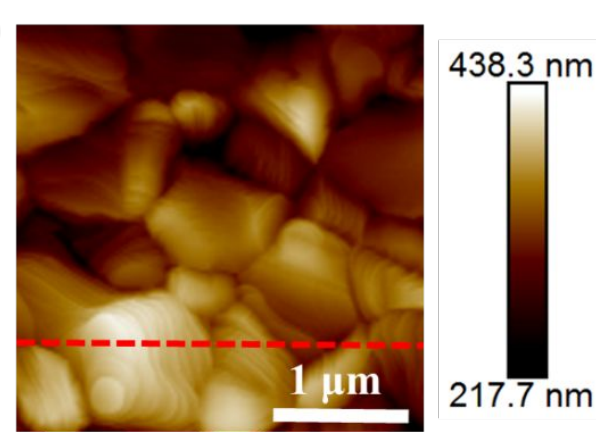

(c)

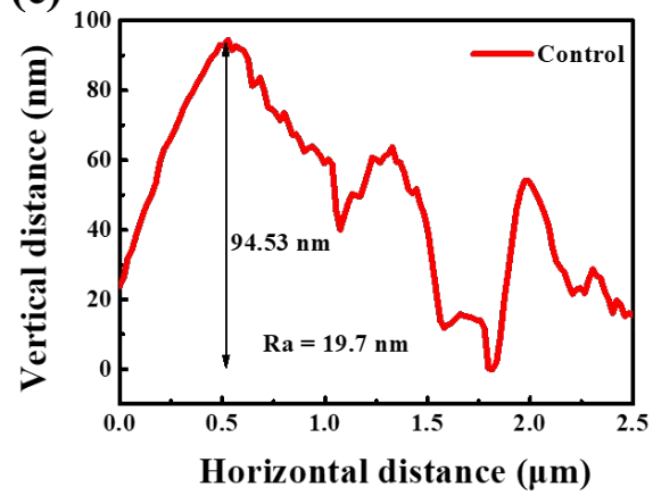

(b)

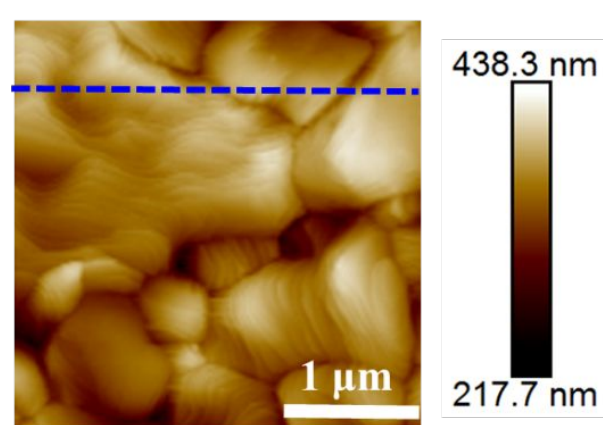

(d)

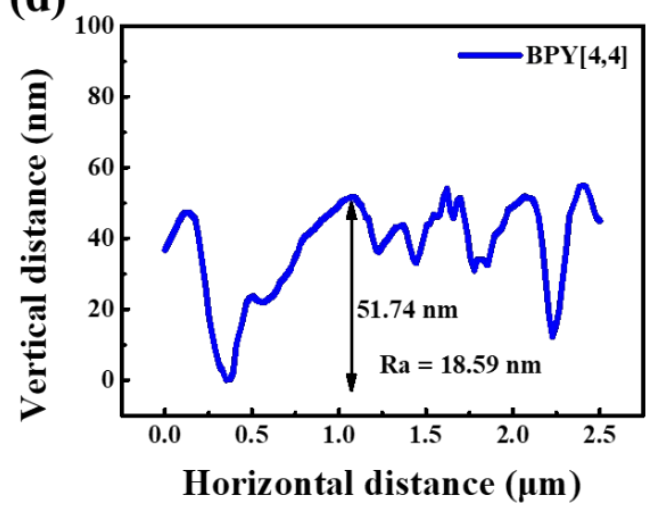

Figure S9. AFM images and surface roughness of $(a, c)$ control film and (b,d) BPY $[4,4]$ added film, respectively. 


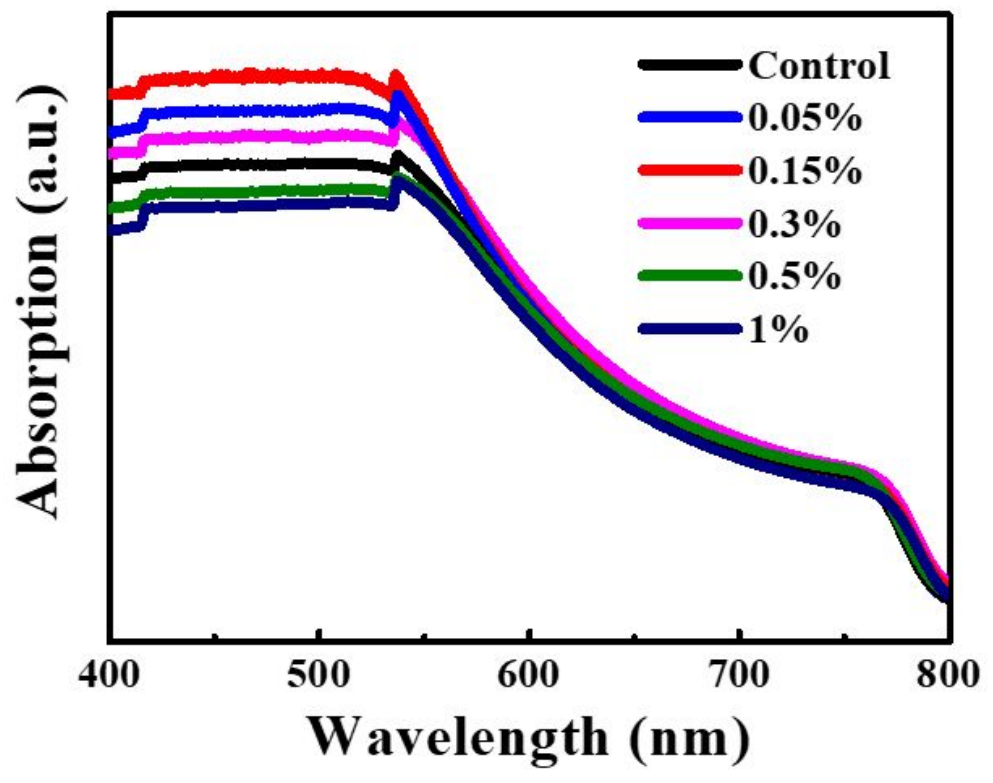

Figure S10. UV-vis absorption spectra of perovskite films containing BPY $[4,4]$ additive with various concentration from 0 to $1 \mathrm{~mol} \%$, respectively. 

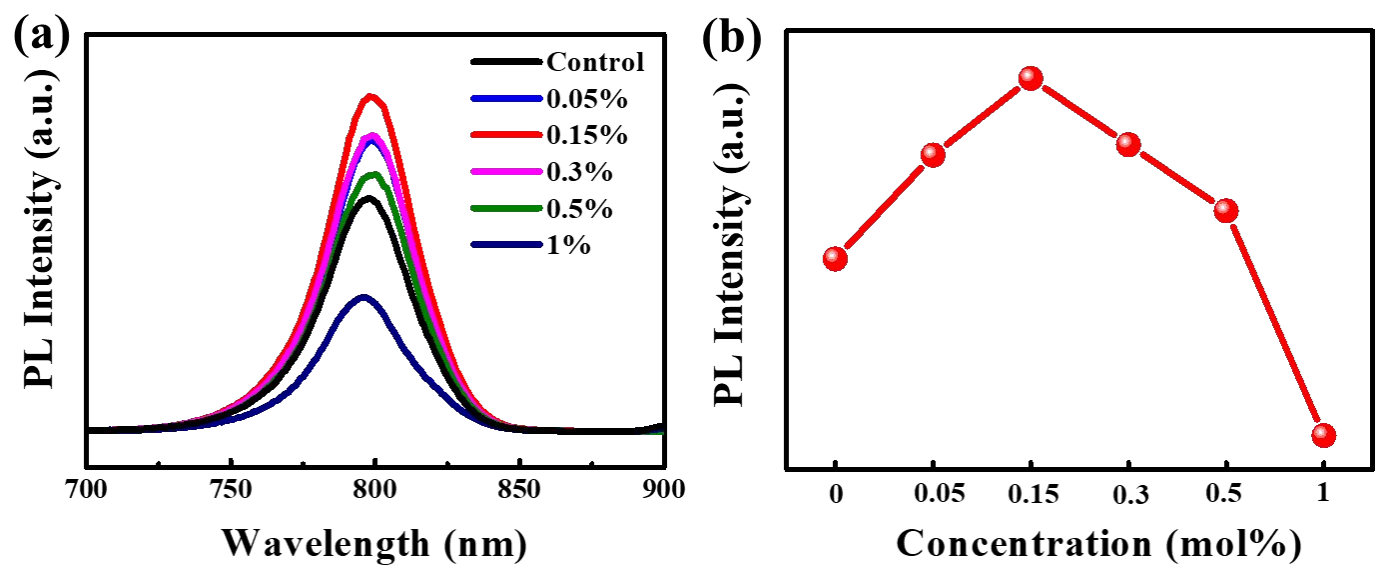

Figure S11. (a) Steady-state PL spectra for perovskite films with BPY $[4,4]$ additive containing various concentration from 0 to $1 \mathrm{~mol} \%$, respectively. (b) The PL peak intensity variation with BPY $[4,4]$ concentration. 
(a)

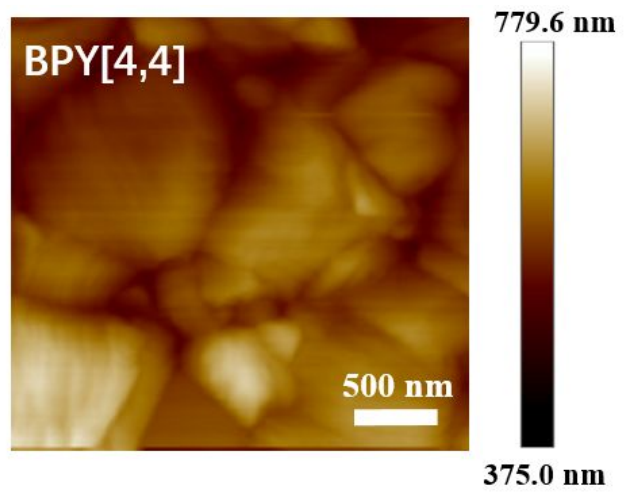

(b)

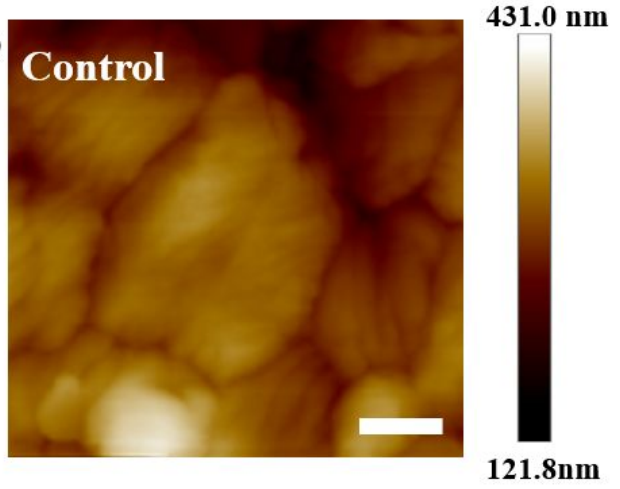

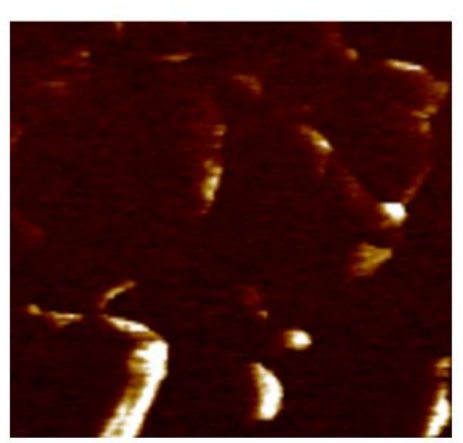

$1.41 \mathrm{pA}$
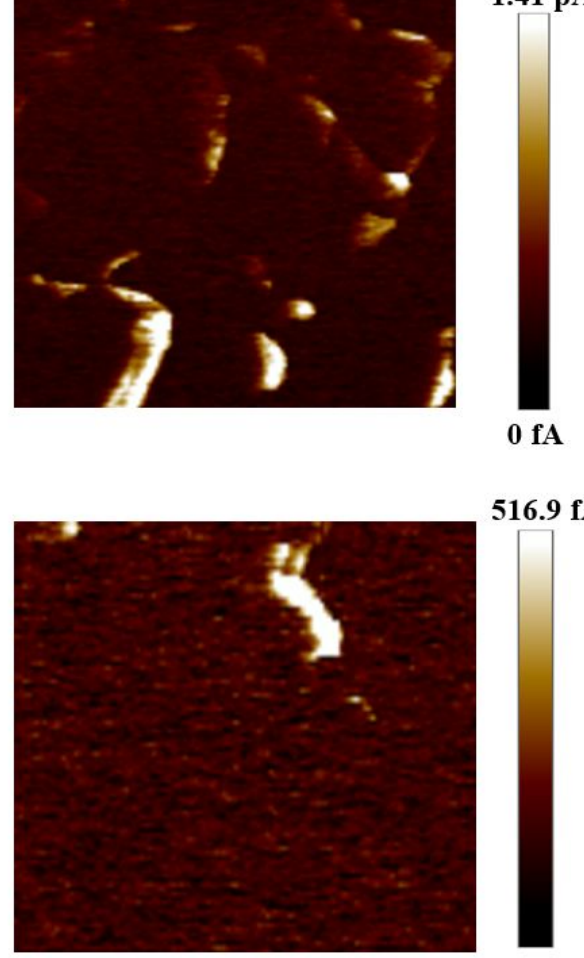

516.9 fA

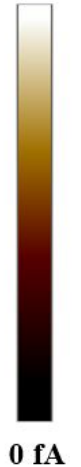

Figure S12. c-AFM images of a) BPY $[4,4]$ and b) control films at $\mathrm{V}_{\text {bias }}=$ $1.0 \mathrm{~V}$. 
(a)

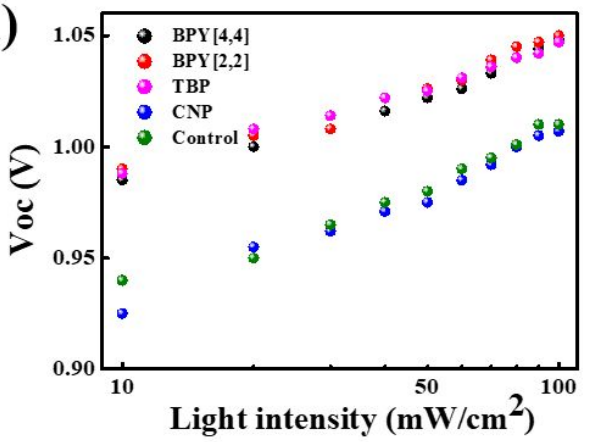

(c)

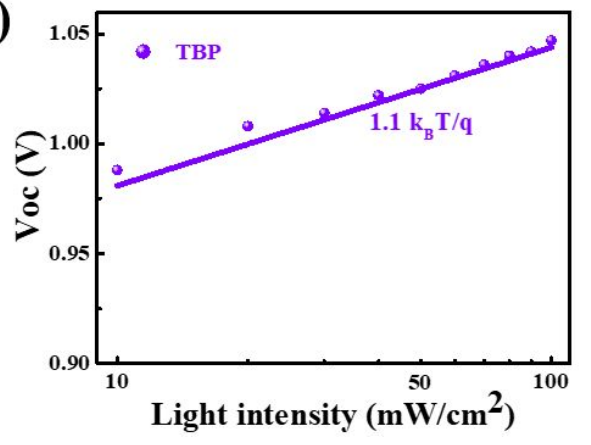

(b)

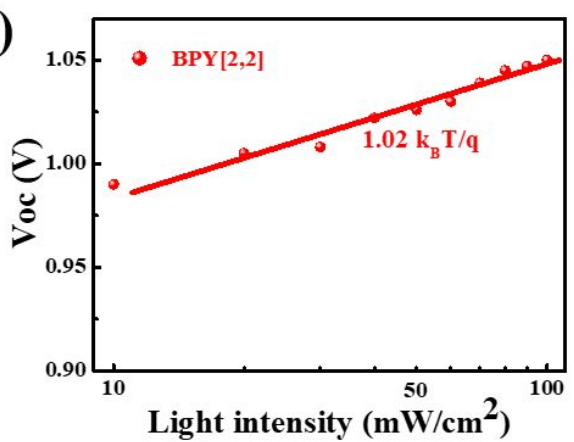

(d)

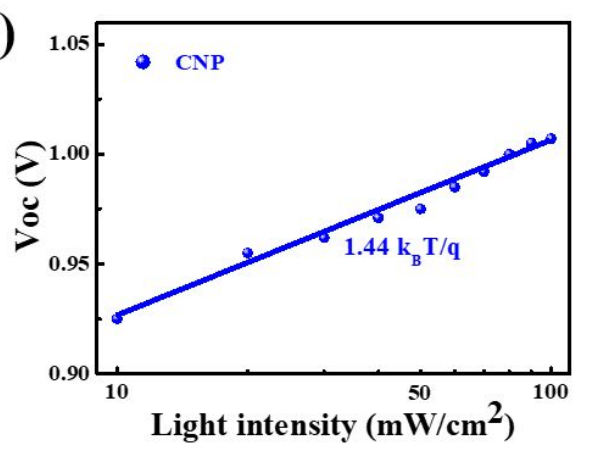

Figure S13. Plots of Voc versus light intensity of the PSCs with different additives, respectively. (a) all (b) BPY[2,2] (c) TBP (d) CNP 
(a)

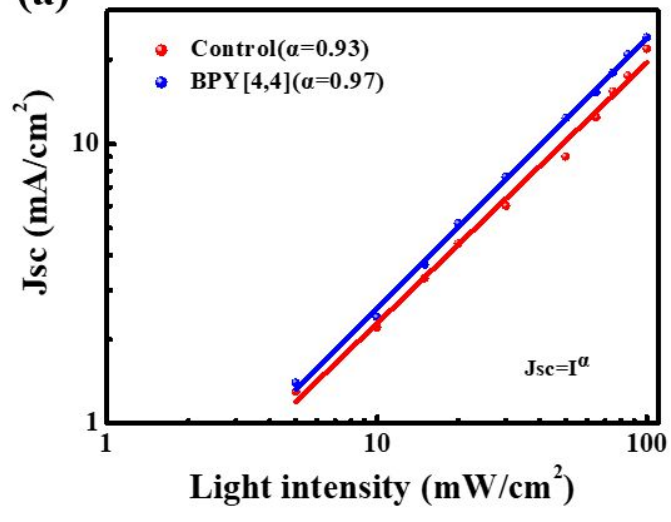

(b)

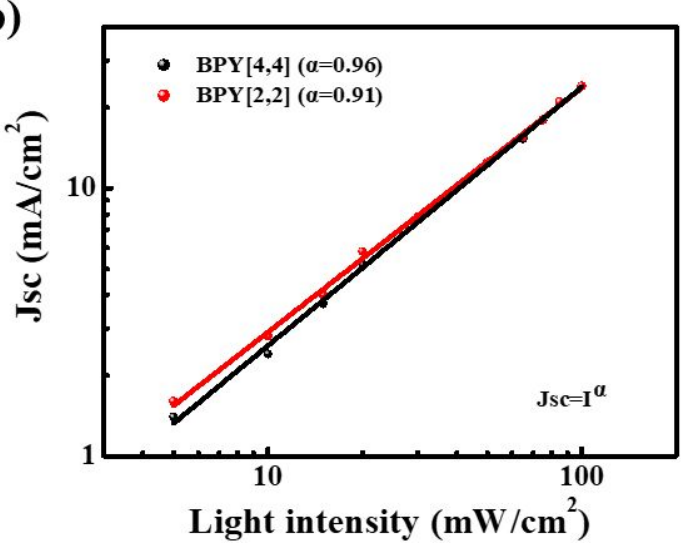

Figure S14. Plots of Jsc versus light intensity of the PSCs. (a) Control and BPY $[4,4](b)$ BPY $[4,4]$ and BPY $[2,2]$ 

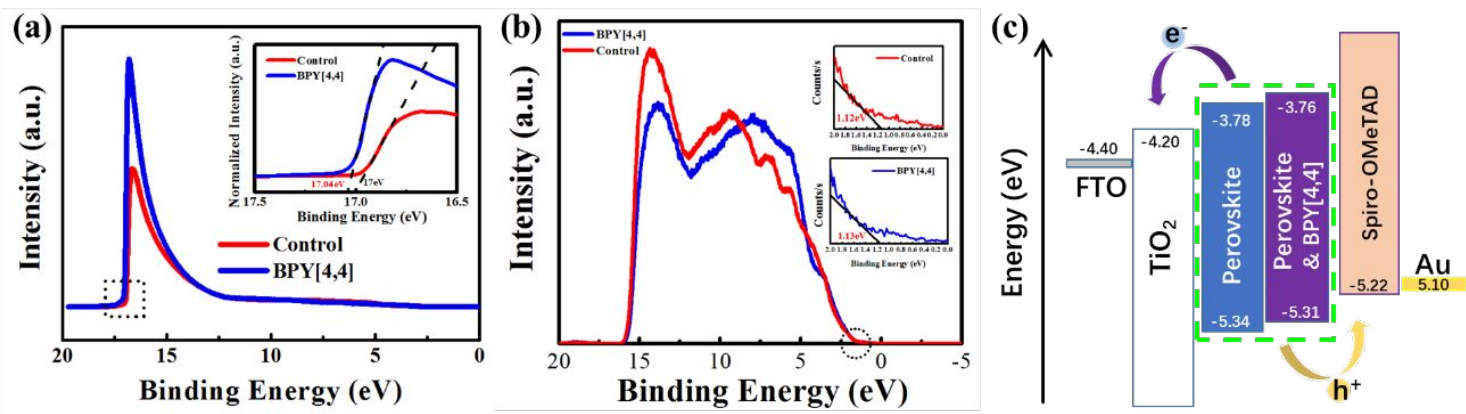

Figure S15. UPS spectra of the control and BPY[4,4] added perovskite films: (a) full spectra with inset photo of cutoff region, and (b) valence band maximum (VBM) region. (c) Schematic energy band diagram and charge injection of the PSCs with or without BPY $[4,4]$ additive. 


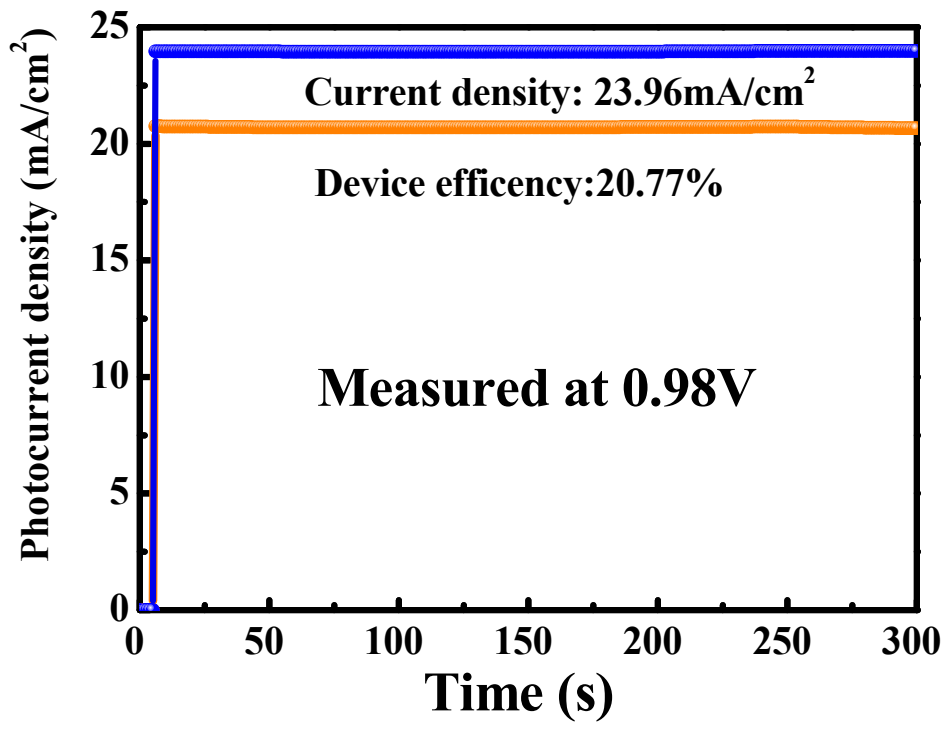

Figure S16. Steady-state current density and PCE for $300 \mathrm{~s}$ at a fixed bias voltage near maximum power point (MPP) about $0.98 \mathrm{~V}$ extracted from the J-V curves for the best efficient PSCs fabricated with $0.15 \mathrm{~mol} \%$ BPY $[4,4]$ added perovskite films. 
Table S1 Comparison of photovoltaic parameters with different additives at $0.15 \mathrm{~mol} \%$ under AM $1.5 \mathrm{G}$ illumination $\left(100 \mathrm{~mW} \mathrm{~cm}^{-2}\right)$.

\begin{tabular}{ccccc}
\hline Additives & $\begin{array}{c}\mathrm{J}_{\mathrm{sc}} \\
{\left[\mathrm{mA} \mathrm{cm}^{-2}\right]}\end{array}$ & $\begin{array}{c}\mathrm{V}_{\mathrm{oc}} \\
{[\mathrm{V}]}\end{array}$ & $\begin{array}{c}\mathrm{FF} \\
{[\%]}\end{array}$ & $\begin{array}{c}\text { PCE } \\
{[\%]}\end{array}$ \\
\hline Control & 22.19 & 1.02 & 81.17 & 18.38 \\
BPY[4,4] & 24.00 & 1.05 & 82.64 & 20.82 \\
BPY[2,2] & 24.01 & 1.07 & 76.07 & 19.53 \\
TBP & 23.90 & 1.06 & 75.37 & 19.09 \\
CNP & 22.63 & 1.00 & 80.20 & 18.12 \\
\hline
\end{tabular}


Table S2 Comparison of photovoltaic parameters for BPY $[4,4]$ added PSCs with different amounts from 0 to 1 mol\% under AM $1.5 \mathrm{G}$ illumination $\left(100 \mathrm{~mW} \mathrm{~cm}^{-2}\right)$.

\begin{tabular}{ccccc}
\hline $\begin{array}{c}\text { BPY }[4,4] \\
\text { concentration } \\
(\mathrm{mol} \%)\end{array}$ & $\begin{array}{c}\mathrm{J}_{\mathrm{sc}} \\
{\left[\mathrm{mA} \mathrm{cm}^{-2}\right]}\end{array}$ & $\begin{array}{c}\mathrm{V}_{\mathrm{oc}} \\
{[\mathrm{V}]}\end{array}$ & $\begin{array}{c}\mathrm{FF} \\
{[\%]}\end{array}$ & $\begin{array}{c}\text { PCE } \\
{[\%]}\end{array}$ \\
\hline 0 & 21.88 & 0.99 & 78.89 & 17.09 \\
0.05 & 22.45 & 1.01 & 77.28 & 17.52 \\
0.15 & 22.60 & 1.02 & 81.51 & 18.78 \\
0.30 & 22.19 & 0.99 & 79.46 & 17.45 \\
0.50 & 22.19 & 0.99 & 77.61 & 17.05 \\
1 & 21.77 & 0.96 & 76.99 & 16.09 \\
\hline
\end{tabular}


Table S3 Summary of PL lifetimes of perovskite films without and with BPY $[4,4]$ treatment deposited on glass substrates, the TRPL decay curves can be well fitted by the decay function: $I(t)=A+B_{1} \exp \left(-t / \tau_{1}\right)+B_{2} \exp \left(-t / \tau_{2}\right)$, where $\tau_{1}$ and $\tau_{2}$ stand for fast and slow decay time constant, respectively, while the $\mathrm{B}$ is the corresponding decay amplitude and $\mathrm{A}$ is a constant for the base-line offset.

\begin{tabular}{ccccccc}
\hline Sample & $\tau_{1}(\mathrm{~s})$ & $\tau_{2}(\mathrm{~s})$ & $\mathrm{A}$ & $\mathrm{B}_{1}$ & $\mathrm{~B}_{2}$ & Average lifetime(ns) \\
\hline Control & 31.4 & 157.2 & 97.1 & 2024.6 & 6315.1 & 126 \\
BPY[4,4] & 31.8 & 246.1 & 239.1 & 1489.6 & 6990.4 & 208 \\
\hline
\end{tabular}


Table S4 Summary of PL lifetimes of perovskite films treated with four additives on glass substrates, the TRPL decay curves can be well fitted by the decay function: $I(t)=A+B_{1} \exp \left(-t / \tau_{1}\right)+B_{2} \exp \left(-t / \tau_{2}\right)$, where $\tau_{1}$ and $\tau_{2}$ stand for fast and slow decay time constant, respectively, while the $\mathrm{B}$ is the corresponding decay amplitude and A is a constant for the base-line offset.

\begin{tabular}{ccccccc}
\hline Sample & $\tau_{1}(\mathrm{~s})$ & $\tau_{2}(\mathrm{~s})$ & $\mathrm{A}$ & $\mathrm{B}_{1}$ & $\mathrm{~B}_{2}$ & Average lifetime $(\mathrm{ns})$ \\
\hline Control & 31.4 & 157.2 & 97.1 & 2024.6 & 6315.1 & 126 \\
BPY[4,4] & 27.5 & 234.9 & 196.8 & 1525.5 & 6472.6 & 188 \\
BPY[2,2] & 26.5 & 192.7 & 174.0 & 1844.4 & 6004.5 & 150 \\
TBP & 11.2 & 161.3 & 118.0 & 2732.0 & 6178.2 & 110 \\
CNP & 2.8 & 198.0 & 48.6 & 1095.8 & 843.5 & 85 \\
\hline
\end{tabular}


Table S5 Statistics of the FWHM values in Figure 3a and Figure S6

\begin{tabular}{cl}
\hline sample & FWHM \\
\hline Control & $0.124^{\circ}$ \\
BPY $[4,4] 145^{\circ} \mathrm{C} 12 \mathrm{~min}$ & $0.185^{\circ}$ \\
BPY $[4,4] 145^{\circ} \mathrm{C} 15 \mathrm{~min}$ & $0.162^{\circ}$ \\
BPY $[4,4] 145^{\circ} \mathrm{C} 18 \mathrm{~min}$ & $0.132^{\circ}$ \\
BPY $[4,4] 155^{\circ} \mathrm{C} 12 \mathrm{~min}$ & $0.112^{\circ}$ \\
BPY $[4,4] 155^{\circ} \mathrm{C} 15 \mathrm{~min}$ & $0.095^{\circ}$ \\
BPY $[4,4] 155^{\circ} \mathrm{C} 18 \mathrm{~min}$ & $0.118^{\circ}$ \\
\hline
\end{tabular}

